Acta Anaesthesiologica

Scandinavica

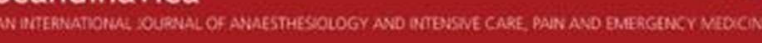

\title{
Management of postoperative pain in Spain: a nationwide survey of practice
}

\begin{tabular}{|r|l|}
\hline Journal: & Acta Anaesthesiologica Scandinavica \\
\hline Manuscript ID & AAS-16-0585.R1 \\
\hline Wiley - Manuscript type: & Clinical investigation \\
\hline Date Submitted by the Author: & n/a \\
\hline Complete List of Authors: & $\begin{array}{l}\text { MoNTES, ANTONIO; Hospital del Mar, ANESTHESIA. PAIN UNIT } \\
\text { aguilar, jose; Son Llatzer Hospital, Anaesthesiology } \\
\text { Benito, Maria del Carmen; Hospital General Universitario Gregorio } \\
\text { Maranon, Anesthesiology } \\
\text { Caba, Fernando; Hospital Universitario de Valme, Anesthesiology } \\
\text { Margarit, Cesar; Hospital General Universitari d'Alacant, Anesthesiology }\end{array}$ \\
\hline Key Words: & $\begin{array}{l}\text { Postoperative acute-pain, Survey, Acute Pain Service, Organizational } \\
\text { change }\end{array}$ \\
\hline Subject area: & \begin{tabular}{l} 
Pain \\
\hline
\end{tabular} \\
\hline
\end{tabular}




\title{
Title page
}

\section{Title: Management of postoperative pain in Spain: a nationwide survey of practice}

\author{
Authors: \\ Antonio Montes \\ Jose Luis Aguilar \\ M Carmen Benito \\ Fernando Caba \\ Cesar Margarit \\ Acute Pain Group of the Spanish Pain Society (SED)
}

Name and location of the institutions

- Antonio Montes

Chairman, Pain Clinic. Department of Anaesthesiology. Parc de Salut MAR. Fundació IMIM. Neurosciences Programme. Perioperative Medicine Research Group. Universitat Autònoma de Barcelona, Barcelona, Spain

- José L Aguilar

Chair Anaesthesia Department and Pain Clinic. Hospital Universitario Son Llatzer. Palma de Mallorca, Spain

- Maria del Carmen Benito 
Staff Anaesthesiologist. Acute Pain Service. Hospital General Gregorio Marañón. Madrid, Spain

- Fernando Caba

Staff Anaesthesiologist. Department of Anaesthesiology. Hospital Nuestra Señora de Valme. Sevilla, Spain

- César Margarit Ferri Chairman, Pain Clinic. Department of Anaesthesiology. Hospital General Universitario de Alicante. Alicante, Spain

Short title: Postoperative pain in Spain Word count (3621)

Corresponding author:

Dr. Antonio Montes Pérez

Department of Anaesthesiology, Parc de Salut MAR. Fundació IMIM. Neurosciences Programme.

Perioperative Medicine Research Group.

Universitat Autònoma de Barcelona, Barcelona, Spain.

Passeig Maritim 25-29, 08003 Barcelona, Spain.

Phone: 0034932483353

FAX: 0034932483617

Email: amontes@parcdesalutmar.cat 


1
2
3
4
5
6
7
8
9
10
11
12
13
14
15
16
17
18
19
20
21
22
23
24
25
26
27
28
29
30
31
32
33
34
35
36
37
38
39
40
41
42
43
44
45
46
47
48
49
50
51
52
53
54
55
56
57
58
60

No relevant conflict of interest

14

15

16

17

19

20

21

22

23

25

26

27

28

30

31

33

34

35

36

37

38

39

40

41

42

44

45

46

47

48

49

51

52

53

54

55

56

57

58

59

60 


\begin{abstract}
Background: Although the need for structured assessment and management of acute postoperative pain has been recognized, practices and responsibilities vary between and within hospitals and countries. We sought to determine current pain management practices in Spanish hospitals with and without acute pain services (APSs) or acute pain management programmes (APMPs) and compare them to practices reported for 1997-1998.

Methods: Members of the Spanish Pain Society and APS/APMP heads were asked to respond to a survey. Responses were stratified by hospital size $(<200$ or $\geq 200$ beds) and APS/APMP presence or not. Categorical variables were described by percentages and the $95 \%$ confidence interval and continuous ones by the median and interquartile range.
\end{abstract}

Results: Responses were received from $42.4 \%$ of hospitals with $\geq 200$ beds (vs. $9.6 \%$ of the smaller ones). We fully analysed only data for the larger hospitals, $57.7 \%$ of which had an APS or APMP. Full-time pain physicians were on staff in $28.6 \%$ of large hospitals; $25 \%$ had full-time nurses. Patients received written information about postoperative pain in $34.8 \%$ of APS/APMP hospitals, and $72 \%$ of them recorded pain assessments routinely. Protocols reflected interdepartmental consensus in $80.8 \%$; training in postoperative pain was organised in 54\%. Respondents thought pain was well or very well managed in 46.4\%. In APS/APMP hospitals the following results had improved: provision of written information for patients $(58.5 \%$ vs. $0 \%)$, the recording of pain 
assessments ( $93 \%$ vs. $43.8 \%$ ), consensus on a pain scale ( $92.5 \%$ vs. $41.9 \%)$, use of protocols ( $99.7 \%$ vs. $55.2 \%)$, analysis of quality indicators $(52.8 \%$ vs. $15.4 \%)$, training ( $73 \%$ vs. $26.9 \%)$, and respondents' satisfaction with pain management in their hospital $(68.6 \%$ vs. $9.5 \%)$.

Conclusions: The presence of an APS or APMP is associated with better results on indicators of quality of acute postoperative pain management. 


\section{Introduction}

Specialist teams to manage postoperative analgesia emerged in the 1970s. ${ }^{1}$ Since then, clinical practice guidelines have been published for the benefit of the many acute pain services (APSs) established, ${ }^{2}$ and good pain control has become an indicator of quality of care and good clinical practice. ${ }^{3}$ How well APSs achieve their goals in most cases of postoperative pain is difficult to determine, however. Since the early 1990s national and international surveys have addressed the question, by sending mail surveys to hospital APSs or individual physicians, even though this approach may overestimate the quality of care. ${ }^{4}$ Meanwhile, many professional associations have promoted the creation of APSs to centralise responsibility for pain control, although several studies have questioned their efficiency ${ }^{5}$ and drawn attention to the many organisational models occluded behind the name these services share ${ }^{6,7}$ Some authors have suggested a new approach—acute pain management programmes (APMPs)—to foster consensus among all hospital staff involved in pain control rather than encourage reliance solely on APS staff. ${ }^{8}$ Yet APSs do seem to have played a role in improving postoperative pain management. Some positive effects are the result of direct interventions on pain through treatments provided, protocols developed, and advanced analgesic techniques applied. Others are indirect, the result of providing patients with appropriate information, training staff to evaluate pain systematically, and periodically evaluating the effectiveness of practice by analysing specific indicators. ${ }^{9,10}$ Recently, nationwide studies of improvements in postoperative pain control over time, particularly those achieved through APSs, 
have appeared. ${ }^{11,12}$ Most agree that APSs have increased in number, but some have called their organisation into question. ${ }^{11}$

A 1997-1998 survey by Puig et al. ${ }^{13}$ analysed the situation of postoperative pain management in Spain after a structured questionnaire was sent to anaesthesia departments at all Spanish hospitals. Our aim was to update our understanding of Spanish postoperative pain management 15 years later (2012) by surveying a representative sample of hospitals with and without APSs or APMPs and compare the results with the situation described earlier. 


\section{Methods}

Sample

All hospitals performing surgery were identified in the 2011 catalogue of Spanish hospitals. ${ }^{14}$ Of the 794 hospitals listed, 537 were performing surgery.

\section{Questionnaire}

Members of the Acute Pain Group of the Spanish Pain Society (SED) designed the questionnaire (Appendix) with a specialised marketing and opinion research company. Twenty-four items with closed answers (some with free-text fields for clarification) and one open question covered the following aspects of pain management or staff organisation. Two questions (Q9b, on the level of APS/APMP coverage in terms of the percentage of patients attended through this approach, and Q11, on whether all or only some patients are so attended) overlapped, such that Q11 served as a way to detect incongruent responses, a way of ascertaining the quality of responses to Q9b. The 24 items covered pain treatments and available human resources, if and when patients were informed about postoperative pain, management protocols in effect and type, whether postoperative pain data were analysed for quality-of-care indicators, staff training (for pain specialists and/or other staff), and the respondents' overall assessment of their hospital's pain management. Members of the Acute Pain Group piloted and revised the questionnaire. Members of SED were then sent three email invitations to answer the final version. Also eligible were heads of APSs or APMPs in hospitals performing surgeries. An APMP was defined as a hospital- 
wide organisational structure to provide information and assess and treat postoperative pain. ${ }^{8}$ APS/APMP heads were also visited individually to ensure they knew about the project and had received an explanatory brochure. The invited respondents accessed the questionnaire with a personal identifier included in their invitation email, but anonymity was maintained by removing names from the database before data extraction. Responses were collected on the SED website between 1 October and 23 November 2012. When we received multiple responses for continuous variables from a single hospital, we analysed the mean scores; when we received multiple responses for categorical variables and it was not possible to establish a correct answer, we eliminated the response. Figure 1 summarises the development and data collection processes started and completed in 2012.

\section{Data analysis}

Data were initially analysed in two hospital groups, those with $<200$ beds $(n=353)$ and those with $\geq 200$ beds $(n=184)$. The larger facilities offered 111106 (69\%) of the 161022 beds available in hospitals performing surgery. We also analysed data according to whether hospitals belonged to the national health service (NHS) or not, were privately run but NHS affiliated, or were entirely private, and whether or not the facility was a teaching hospital. Finally, data were stratified according to whether a hospital had an APS or APMP or not.

The SPSS statistical package (version 20.0 for Windows) was used. Continuous variables were described by the median and interquartile range 


\begin{abstract}
because we observed nonnormal distributions. Categorical variables were described by percentages and the $95 \%$ confidence interval for the designated hospital type. To compare categorical variables, we used the chi-square test or, if frequencies below 5 for variables in $2 \times 2$ contingency tables were foreseen, we used the Fisher exact test. Statistical significance was set at 0.05 .
\end{abstract}




\section{Results}

Returned questionnaires

One hundred forty-four completed questionnaires from 112 hospitals were returned; we received multiple responses from 22 centres and achieved an overall response rate of $20.9 \%$. The rates were $42.4 \%$ for hospitals with $\geq 200$ beds and $9.6 \%$ for smaller hospitals (Figure 1). Given the low response rate from smaller hospitals, results for these facilities should be interpreted with caution; therefore, the analysis of APS/APMP activity in most tables reflects only the situation in large hospitals. A single case of disagreement between two respondents to a categorical question (Q22) was received from one hospital; that hospital's response was therefore discarded. Most of the hospitals with $\geq 200$ beds were publicly owned NHS facilities ( $87.2 \%), 6.4 \%$ were privately owned NHS-affiliated facilities, and $6.4 \%$ were unaffiliated private hospitals. In contrast, $47.1 \%$ of the smaller hospitals were public, $35.3 \%$ were private affiliated facilities, and $17.6 \%$ were entirely private. The response rate among public NHS hospitals of $\geq 200$ beds was $44.4 \%$, indicating that the sample could be considered representative of this hospital type (which covers $74.6 \%$ of the surgical caseload in Spain). The proportion of large hospitals offering residency training programmes was $83.3 \%$; in contrast, only $20.6 \%$ of hospitals with $<200$ beds were teaching hospitals.

Because the open question Q20h, about analgesics and analgesic techniques, required answers in several free-text fields (Appendix), the 
responses were highly varied. Statistical analysis was considered too complex for this article, so it will be reported or reassessed at a later date.

Surgical caseload, pain management, and human resources

Table 1 shows surgical activity according to hospital size, surgical complexity/patient hospitalisation category, and surgical specialty. An APS or APMP was present in $57.7 \%$ of hospitals with $\geq 200$ beds (Table 2 ). Table 3 shows the scope of APS/APMP activity. In nearly $40 \%$ of large hospitals, these services were attending over $70 \%$ of operated patients. Nonsurgical acute pain was also managed by these services in over $16 \%$ of the hospitals in this category.

Protocols were in effect in the majority of the large hospitals and in nearly all APS/APMP hospitals (vs. slightly over half of those without services, $P<0.001$ ) (Table 4). Protocols were based on consensus with the surgical department in nearly $80 \%$ of APS/APMP hospitals. Nearly all Ilarge hospitals reported that their protocols named levels of pain expected (e.g. mild, moderate, severe), but many also established special protocols linked to particular procedures (e.g. for total knee arthroplasty, thoracotomy, laparoscopic cholecystectomy).

Informing patients and recording pain intensity

Patients received written preoperative information about postoperative pain in $34.8 \%$ of the hospitals. Likewise, in 2012 more hospitals (58.5\% of APS/APMP facilities) gave written information (vs. none of the hospitals without specialist 
services), usually during the preanaesthesia evaluation (91.7\%). Other moments when this information was provided were during the patient's presurgical office visit with the surgeon $(4.2 \%)$, or on the ward or in the outpatient surgical area $(4.2 \%)$

Over $70 \%$ of the hospitals with $\geq 200$ beds recorded pain assessments, over $65 \%$ using a specific place in the patient's chart (Table 5). These percentages were significantly higher in APS/APMP hospitals. Pain was recorded in the same section as vital constants in over $85 \%$ of hospitals without a specialist service and in a different, pain-specific document in over half the APS/APMP hospitals. Records were computerised in nearly three quarters of APS/APMP hospitals (vs. only a third of hospitals without a service). Over $70 \%$ of all the large hospitals used standardised scales for recording pain; that percentage was also higher in APS/APMP hospitals. The tools most commonly named were visual analogue scales $(77.9 \%)$, verbal numerical scales $(54.7 \%)$, verbal categorical scales $(24.2 \%)$, and face scales (24.2\%). Over $95 \%$ used the face scales with children and $43.5 \%$ used them with the elderly (43.5\%). Only hospitals with an APS/APMP designated time intervals for assessing pain: in $66.7 \%$ assessments took place every 8 hours, in $23.3 \%$ every 24 hours, in $6.7 \%$ every 4 hours, and in $3.3 \%$ every 6 hours.

Slightly over half (51.9\%) of the hospitals specified a level of pain intensity that obliged use of a rescue analgesic or notification of the patient's physician. On the verbal numerical scale that level was 3 in $26.7 \%$ of the hospitals and 4 in $26.7 \%$. On the verbal categorical scale the level was "moderate" in $78.6 \%$. 
Analysis of quality-of-care indicators, provision of training, and respondents' assessment of performance

Patient records were analysed to suggest ways to improve care in $53.2 \%$ of the hospitals $(75.0 \%$ of APS/APMP hospitals and in $23.1 \%$ in hospitals without either, $P<0.001)$. Specific quality indicators (whether of process or outcomes) were designated in $37.1 \%$ of the hospitals $(52.8 \%$ of APS/APMP hospitals and $15.4 \%$ of those without one, $P=0.003)$.

Training was organised in over half the large hospitals (and in nearly three quarters of APS/APMP hospitals) (Table 6). Sessions usually targeted nurses or anaesthetists and were given by APS/APMP or anaesthesiology department staff. The training was accredited in $67.7 \%$ of the hospitals.

Table 7 shows how well the respondents thought postoperative pain was being managed in their hospital. Overall nearly half thought pain was well or very well managed. Positive evaluations were more frequent in APS/APMP hospitals; fewer than $10 \%$ of hospitals without such services earned high assessments.

Figure 2 compares the 2012 findings to the most important ones reported for $1997-1998 .^{13}$ The only variable that had not clearly improved by 2012 was staffing. However, respondents in the earlier survey were asked about specialists working either part- or full-time in 1997-1998 whereas our survey inquired about full-time specialists. Still we note that more of the hospitals had specialist doctors and nurses available at that time. 
Once the results were analyzed, we did a further post-hoc comparison to detect possible differences between two levels of large hospital: those with 200 to 600 beds and those with $>600$. We found that $65.7 \%$ of Spain's very large hospitals (>600 beds, all of them teaching facilities) had an APS or APMP (vs. $51.2 \%$ of medium-large hospitals, $P<0.05)$. Human resources also differed in these two large hospital groups. Very large hospitals had significantly more physicians assigned full-time to pain management $(35.3 \%$ vs. $23.1 \%$ in mediumlarge hospitals); very large hospitals also had more nurses so assigned (34.2\% vs. $17.4 \%$, respectively) ( $P<0.05$, both comparisons). Conversely, signifcantly fewer very large hospitals assigned their physicians to pain management on a part-time basis ( $24.0 \%$ vs. $43.5 \%$ of medium-large ones); this pattern was the same for nurse assignments (38.2\% vs. $66.3 \%$, respectivamente) $(P<0.05$, both comparisons). There were no significant differences for other responses. 


\section{Discussion}

This survey of postoperative pain management practices in Spain reveals that much has improved since the publication of a survey carried out in 1997$1998,{ }^{13}$ suggesting progress in these years of APS or APMP presence. Fifteen years earlier, postoperative pain management was judged inadequate: most patients received no preoperative information on pain, acute pain was not routinely assessed, and clear chains of responsibility for pain treatment were not widely established. Several ways to improve emerged from the previous study. One suggestion was to encourage collaboration between professional associations of surgeons, nurses, and anaesthetists to develop practice guidelines. Training modules on pain pathophysiology and treatment for medical and nursing students and residents were also suggested. More resources to improve postoperative pain management were called for. Only some of these proposals have been implemented, however, and we found that although more Spanish hospitals have APSs or APMPs, fewer specialist physicians and nurses have been specifically assigned to them.

In spite of progress made, we saw that our quality indicators do not match those reported for other countries, ${ }^{15}$ yet Spain's increase in APS/APMP hospitals (57.7\% in 2012 vs. $29.8 \% 15$ years ago) does follow the trend seen in the United States, ${ }^{16}$ the United Kingdom, ${ }^{17}$ the Netherlands, ${ }^{12}$ and Canada. ${ }^{18}$ In contrast, Denmark first saw an overall rise in hospitals with services between 2000 and 2006 , but found the number had fallen in 2009 , even while accelerated postoperative recovery programmes continued to increase. ${ }^{19}$ Hospital type 
influenced resources in Denmark: university teaching hospitals maintained their APSs in 2009 while the number of services in local and regional hospitals fell significantly. One hypothesis is that APS models may be more feasible in large centres. The results of our post-hoc analysis of our data allowing us to compare Spain's 2012 situation to the Danish one are consistent that hypothesis: the very large Spanish hospitals ( $>600$ beds), all of which were univeristy teaching facilities, had more organized means for managing postoperative pain and also higher percentages of physicians and nurses assigned to carry out the work. In both Spain and Denmark, then, the traditional APS model tends to survive in larger hospitals, while it tends to decrease in smaller hospitals (Spain) or disappear from them (Denmark).

Nonetheless, even though APSs and APMPs have become more numerous in Spain, full-time staff assignments have declined overall: the proportion of APSs with full-time physicians fell from $48 \%$ in $1997-1998$ to $28.6 \%$ in 2012 (nursing staff from $27.9 \%$ to $25 \%$ ). A recent German audit revealed the same trend. ${ }^{11} \mathrm{~A}$ concern is that we found that no assignments at all had been made in $6.8 \%$ of the large Spanish hospitals that reported having an APS: it seems that some services have only been established administratively, given that they have been left unstaffed. A similar situation was reported in 2008 for France, where $72.4 \%$ of hospitals had a postoperative pain quality monitoring programme but only $14.5 \%$ had assigned personnel. ${ }^{20} \mathrm{~A}$ concern is that this situation may mean that the quality of pain management has fallen for some patients. ${ }^{11,22,23} \operatorname{In}$ a substantial minority (37.8\%) of Spanish APS/APMP hospitals, respondents 
estimated that their services are managing only $70 \%$ of the surgical patients. Patients with the most intense pain have often undergone procedures that are seemingly less painful, ${ }^{21}$ possibly because pain specialists are sometimes assigned only to patients after surgeries expected to cause significant pain. An implication is that specialist involvement should not be restricted to patients already routinely treated with more sophisticated analgesic techniques. ${ }^{10}$ In summary, we feel that staffing practices must be examined further because there is observational evidence that intense, ongoing postoperative pain is one of the factors that predict the development of chronic postsurgical pain. ${ }^{24-26}$ Therefore, optimal pain management is essential if we are to prevent acute postoperative pain from becoming chronic. ${ }^{24-26}$

While written information on postoperative pain was given in more hospitals overall in 2012 (34.8\%, up from $30.6 \%$ in $1997-1998),{ }^{13}$ practice in hospitals with or without a specialist service differed greatly: nearly $60 \%$ of the APS/APMP hospitals informed patients but none of those without a specialised approach did so. Expert consensus continues to promote the value of discussing pain assessment, the techniques used to control it, and their possible adverse effects, with emphasis on the advantages of adequate treatment. ${ }^{27}$

Pain was routinely recorded in twice as many hospitals in 2012 as in 19971998. ${ }^{13}$ Regular assessment was significantly higher in APS/APMP hospitals, where interdepartmental consensus on which pain scale to use was also more likely. These findings are consistent with reports that a specialised approach to pain increases staff awareness and improves how pain is assessed and 
recorded. ${ }^{28,28}$ Our finding that slightly more than half of the hospitals with specialised services continued to use separate records, may be attributable to the pain specialists' lack of access to other departments' records. Only $65 \%$ of the hospitals overall designated a specific place for pain assessments in patient charts. Since electronic records are now widespread, we think it is time for hospitals to reflect on whether postoperative pain records should be included with other clinical records so pain can be consistently analysed as a quality-ofcare indicator. $^{3}$

Proactive pain management protocols were followed in nearly all APS/APMP hospitals in 2012 (vs. about half those without one and $80 \%$ of the sample overall). The protocols were mainly based on the foreseen level of pain intensity, as recommended. ${ }^{30}$ However, many hospitals also have procedurespecific analgesic protocols, an option also supported by a systematic review of the literature. ${ }^{31}$ Adherence to multidisciplinary protocols was shown to reduce hospital stays and the number of complications in a meta-analysis of randomised controlled trials, ${ }^{32}$ yet a multicentre survey of 746 hospitals in 7 southern and central European countries found that only $36 \%$ of them had written protocols for postoperative pain. ${ }^{33}$

We found that pain management data were about three-fold more likely to be analysed to plan improvement strategies in hospitals with specialist teams $(75 \%)$ than in those without teams $(23.1 \%)$. Over half of Spain's APS/APMP hospitals were analysing specific quality indicators in 2012 (vs.only about $15 \%$ of other hospitals). Such analysis serves to improve the treatment of postoperative 
pain in a particular hospital ${ }^{34}$ and provides data for benchmarking and new clinical guidelines. ${ }^{11}$

Pain management training sessions were organized in over half the large hospitals in 2012 (vs. $<40 \% 15$ years earlier ${ }^{13}$ ). Hospitals with an APS or APMP-73\% of which offered courses in 2012-account for most of the increase. Spanish training mainly targeted anaesthetists and nurses, in contrast with courses in the Netherlands ${ }^{12}$ and Italy, ${ }^{35}$ where nurses do not usually attend. Because adequate, ongoing training favours better postoperative pain management, ${ }^{36,37}$ it has been suggested that education and training should be available to other caregivers besides anaesthetists. ${ }^{11}$

Our respondents believed pain was well or very well managed in just under half $(46.4 \%)$ of the hospitals, a larger proportion than 15 years earlier $(28.6 \%){ }^{13}$ A favourable evaluation was much more common in APS/APMP hospitals $(68.6 \%)$ than in those without one $(9.5 \%)$.

One limitation of our study is that we cannot rule out sampling bias, given that the response rate for hospitals with $\geq 200$ beds $(42.4 \%)$ was lower than the rate of $53.0 \% 15$ years earlier. ${ }^{13}$ Our response rate for large Spanish hospitals is considered adequate for this type of study, for which methodologists set a sampling threshold of around $20 \%{ }^{38,39}$ However, as we targeted responses from all hospitals performing surgery in Spain, not a sample of them, we therefore recognize the possibility that are results are biased in favour of hosptials with greater interest in postoperative pain control. The response rate for small hospitals (9.6\%) was also lower than the one obtained in the 1997-1998 survey 
(19\%), even though we visited the physicians responsible for pain control in all hospitals in an attempt to improve interest and response. Low response rates in small hospitals may be due to the fact that they are mostly prívate affiliated facilities or entirely prívate ones $(52.9 \%)$, whereas only $12.8 \%$ of the hospitals with $\geq 200$ beds are private facilities. In most small Spanish hospitals, physicians are not full-time staff; as result, they participate relatively little in tasks (such as audits) that do not directly involve patient care. These speculations lead us to surmise that these hospitals have few, if any, structures in place for managing postoperative pain. We cannot, however, be sure of this interpretation because of the low response rate, so we have drawn conclusions about pain management only in large, usually public or mixed-use hospitals. A second limitation is that patients were not directly asked about postoperative pain. Therefore, we are unable to provide evidence that suggests a causal relationship between process indicators (e.g., human resources, patient information, protocols for assessment and treatment, analysis of indicators, training, etc.) and outcomes from the patient's point of view (reduced pain and satisfaction with treatment).

A strength of our survey is that respondents were heads of APSs or APMPs. Surveys of heads of anaesthesiology departments ${ }^{11,13}$ run the risk of recording the views of physicians who might not always be highly familiar with all aspects of postoperative pain management.

In summary, more hospitals had APSs or APMPs in 2012 than 15 years earlier but proportionally fewer specialised physicians and nurses assigned to them. Our findings show better evaluations on the following process indicators in 


\begin{abstract}
APS/APMP hospitals: the provision of written preoperative information about postoperative pain, the recording of pain assessments, consensus on which scale or scales to use consistently throughout the hospital, the establishment of treatment protocols, the study of quality indicators, the availability of training in pain management, and the staff's satisfaction with their hospital's pain management.
\end{abstract}

The Acute Pain Group of the SED plans to carry out a new audit of the APS/APMP within 2 to 4 years. The methodology will be rethought to increase the response rate, particularly in small, private hospitals. We feel it is necessary to deepen our understanding of acute postoperative pain is being treated. We also plan to prioritize obtaining data from patients through structured interviews in order to explore congruence between process and outcome indicators. Full understanding of this situation is necessary for approaching health authorities to obtain greater support for structures that can improve quality treatment for acute pain. 


\section{Acknowledgements}

This research received funding from the Spanish Pain Society (SED) to cover costs. Demométrica in Madrid, Spain, assisted the authors in developing the digital form of the questionnaire. The authors thank Mary Ellen Kerans, M.A. (Freelance Editor, Barcelona, Spain), who translated a version of the manuscript and advised on English usage. 


\section{Figure legend}

Figure 1 Flow chart showing the development of the questionnaire and the data collection process. Abbreviations: APS $=$ acute pain service; APMP $=$ hospitalwide acute pain mangement programme; SED = Spanish Pain Society .

Figure 2 Comparison of findings from the $1997-1998^{13}$ and 2012 surveys. APMPs were introduced into some hospitals around 2007.8 In the earlier study, all pain specialists were working in APSs. Abbreviations: APS = acute pain service; APMP = hospital-wide acute pain mangement programme; $\mathrm{NC}=$ data not collected. 
Figures and tables

\section{(Figure 1)}

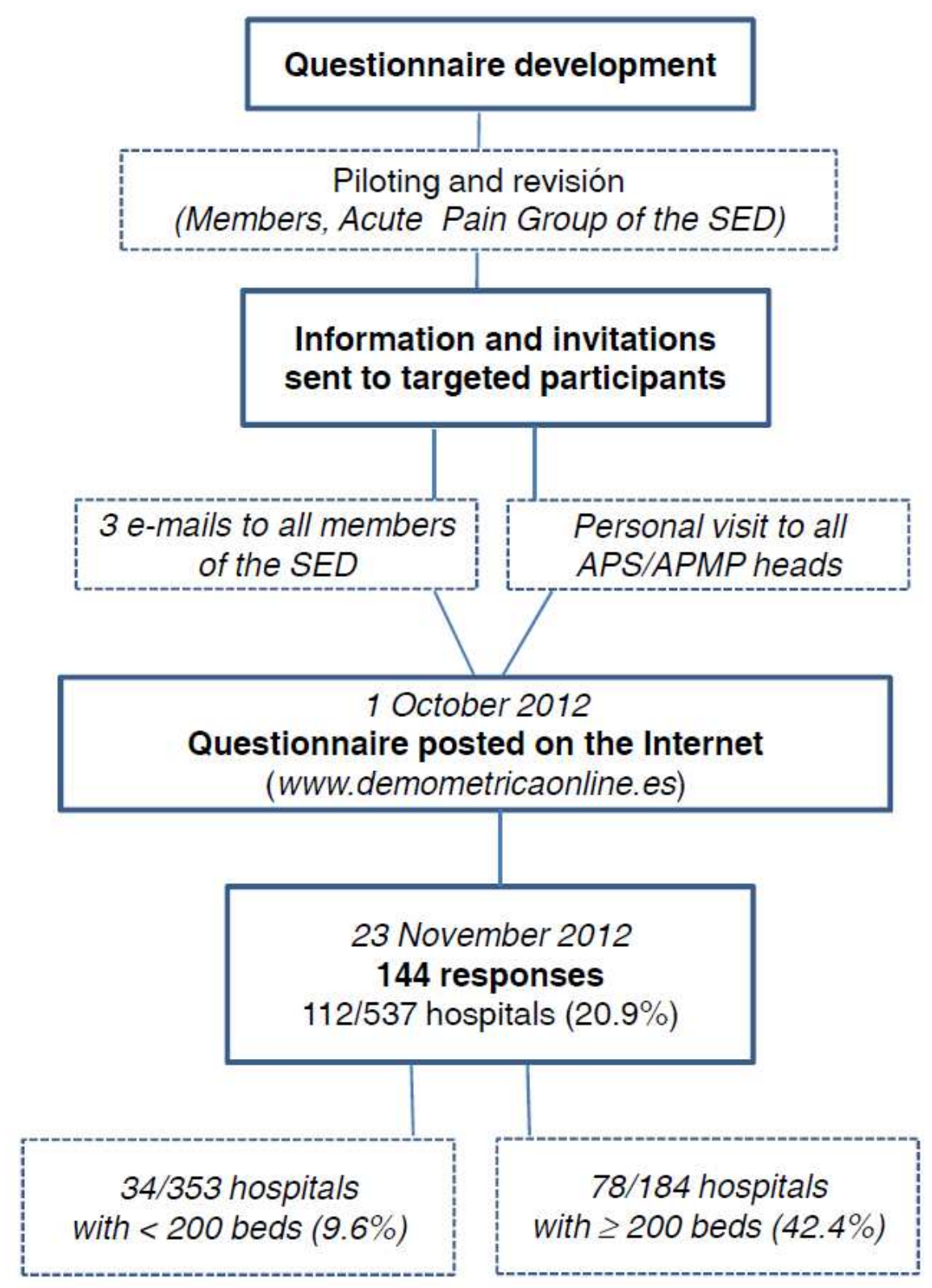




\section{(Figure 2)}

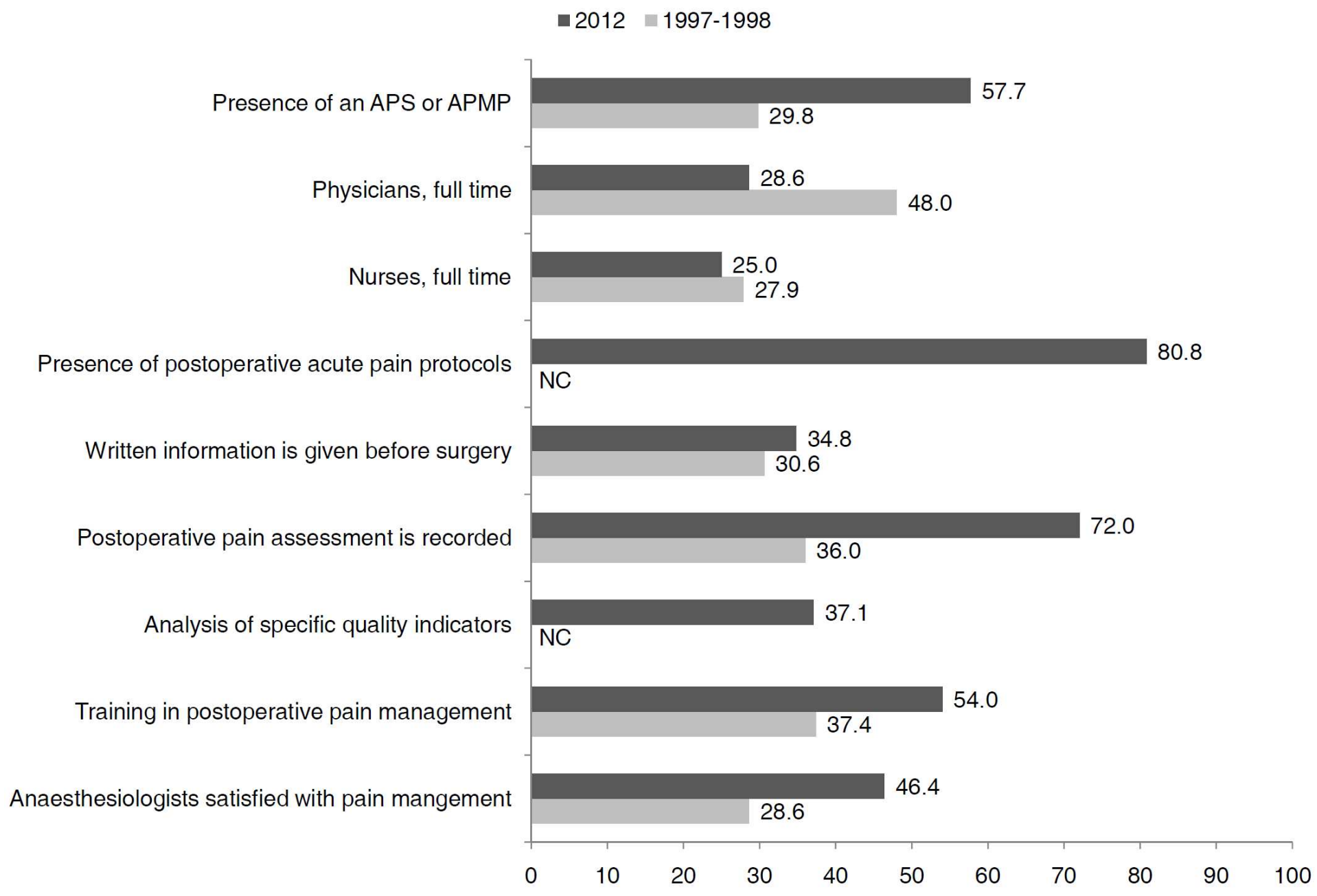


Table 1 Surgical caseloads: Number of procedures each week by hospital size, complexity, patient hospitalisation category, and surgical specialty

\begin{tabular}{|c|c|c|c|c|c|c|}
\hline & \multicolumn{2}{|c|}{$\begin{array}{l}<200 \text { beds } \\
n=34 \\
\text { Median (IQR) }\end{array}$} & \multicolumn{2}{|c|}{$\begin{array}{l}\geq 200 \text { beds } \\
n=78 \\
\text { Median (IQR) }\end{array}$} & \multicolumn{2}{|c|}{$\begin{array}{l}\text { Total } \\
\mathrm{n}=112 \\
\text { Median (IQR) }\end{array}$} \\
\hline Level of complexit & $S$ & & & & & \\
\hline Hospitalised & 37.5 & $(20.0-80.0)$ & 100.0 & $(75.0-185.0)$ & 100.0 & $(40.0-149.0)$ \\
\hline Major outpatient & 35.0 & $(20.0-65.0)$ & 70.0 & $(30.0-115.0)$ & 58.0 & $(25.0-100.3)$ \\
\hline Minor outpatient & 35.0 & $(17.3-80.0)$ & 50.0 & $(30.0-97.8)$ & 50.0 & $(25.0-83.8)$ \\
\hline Total & 126.0 & $(65.0-232.5)$ & 250.0 & $(142.5-410.0)$ & 171.5 & $(100.0-367.0)$ \\
\hline Surgical specialty & & & & & & \\
\hline
\end{tabular}




\begin{tabular}{|c|c|c|c|c|c|c|}
\hline General & 20.0 & $(11.5-40.0)$ & 32.0 & $(20.0-50.0)$ & 30.0 & $(20.0-50.0)$ \\
\hline Orthopaedic & 30.0 & $(20.0-40.0)$ & 30.0 & $(20.0-52.0)$ & 30.0 & $(20.0-50.0)$ \\
\hline Gynaecologic & 10.0 & $(6.0-20.0)$ & 20.0 & $(12.0-30.0)$ & 17.0 & $(10.0-30.0)$ \\
\hline Urologic & 8.0 & $(5.0-20.0)$ & 15.0 & $(10.0-24.5)$ & 13.5 & $(8.0-24.0)$ \\
\hline Plastic & 4.0 & $(0.0-17.5)$ & 8.0 & $(0.0-15.0)$ & 6.0 & $(0.0-15.0)$ \\
\hline Ear-nose-throat & 8.0 & $(4.0-10.0)$ & 15.0 & $(10.0-20.0)$ & 12.0 & $(8.0-20.0)$ \\
\hline Vascular & 4.0 & $(0.0-8.5)$ & 9.0 & $(2.3-15.0)$ & 7.0 & $(0.0-13.0)$ \\
\hline Maxillofacial & 0.5 & $(0.0-5.3)$ & 4.0 & $(0.0-13.5)$ & 3.0 & $(0.0-12.0)$ \\
\hline Cardiac & 0.0 & $(0.0-0.0)$ & 0.0 & $(0.0-8.0)$ & 0.0 & $(0.0-6.0)$ \\
\hline Neurosurgery & 0.0 & $(0.0-5.0)$ & 5.0 & $(0.0-11.0)$ & 2.0 & $(0.0-10.0)$ \\
\hline Thoracic & 0.0 & $(0.0-1.3)$ & 2.0 & $(0.0-8.0)$ & 0.0 & $(0.0-5.0)$ \\
\hline
\end{tabular}

Abbreviation: $I Q R=$ interquartile range 
Table 2 APS or APMP presence and human resources assigned according to postoperative pain management

\begin{tabular}{|c|c|c|c|}
\hline & $\begin{array}{l}<200 \text { beds } \\
n=34\end{array}$ & $\begin{array}{l}\geq 200 \text { beds } \\
n=78\end{array}$ & $\begin{array}{l}\text { Total } \\
n=112\end{array}$ \\
\hline & $\%^{*} \quad(95 \% \mathrm{Cl})$ & $\%^{*} \quad(95 \% \mathrm{Cl})$ & $\%^{*} \quad(95 \% \mathrm{Cl})$ \\
\hline APS or APMP & $44.1(27.4-60.8)$ & 57.7 (44.7-66.7) & $53.6(44.4-62.8)$ \\
\hline Physicians, full-time & $13.3(0.0-31.1)$ & $28.6(14.9-42.3)$ & $24.6(13.4-35.8)$ \\
\hline Physicians, part-time & $53.3(28.1-78.5)$ & $56.1(40.9-71.3)$ & $55.4(42.4-68.4)$ \\
\hline Nurses, full-time & $20.0(0.0-40.2)$ & $25.0(12.2-37.8)$ & $23.7(12.8-34.6)$ \\
\hline Nurses, part-time & $20.0(0.0-40.2)$ & $31.8(18.0-45.6)$ & $28.8(17.2-40.4)$ \\
\hline No specialised staff & $26.7(4.3-49.1)$ & $6.8 \quad(0.0-14.2)$ & 11.9 (3.6-20.2) \\
\hline
\end{tabular}

Abbreviations: APS = acute pain service; APMP = hospital-wide acute pain management programme; $\mathrm{Cl}=$ confidence interval. 
Table 3 Scope of APS or APMP care, by hospital size

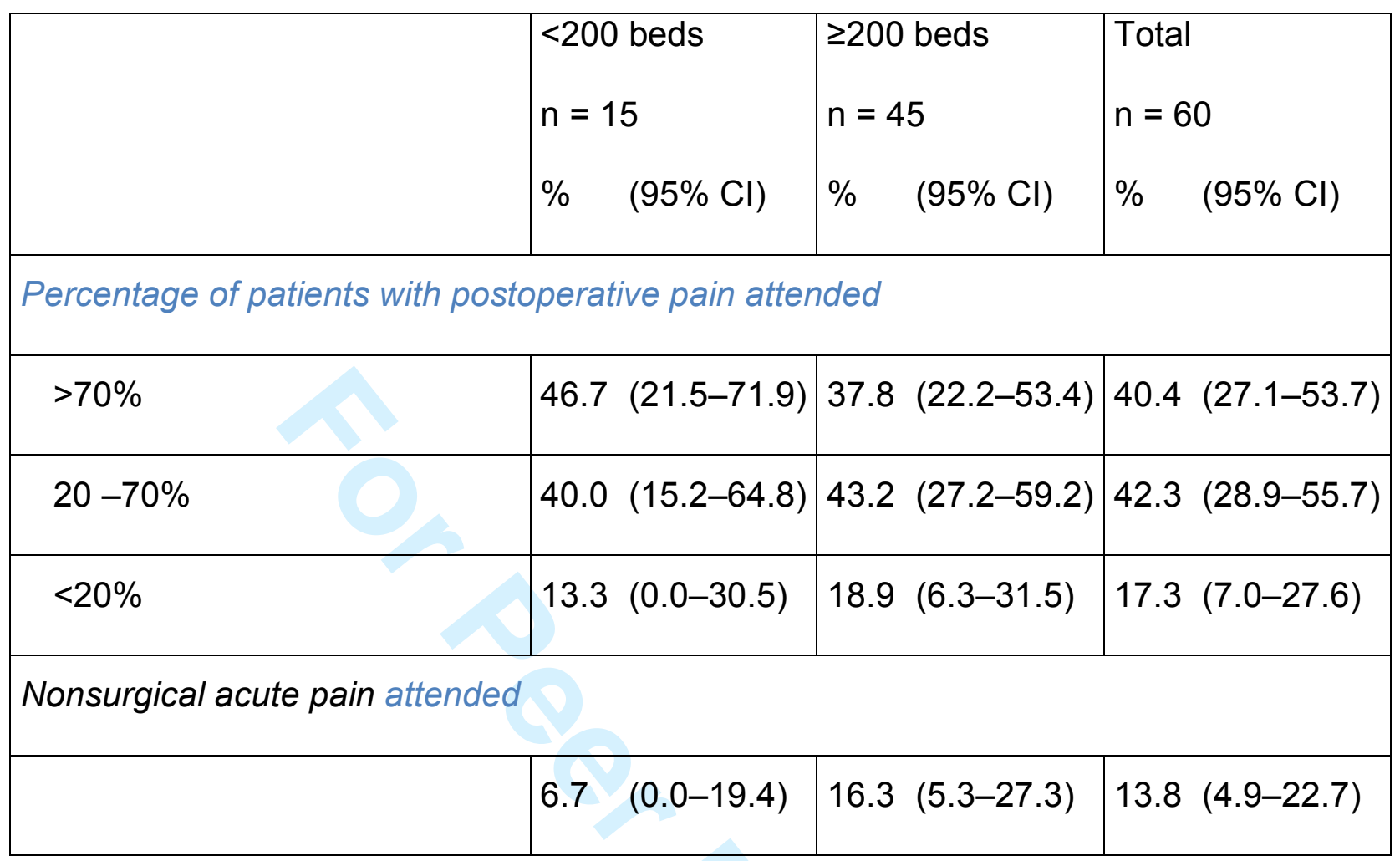

Abbreviations: APS = acute pain service; APMP = hospital-wide acute pain management programme; $\mathrm{Cl}=$ confidence interval. 
Table 4 Presence of protocols and how they are used in hospitals with $\geq 200$ beds and according to the presence or absence of an APS or APMP

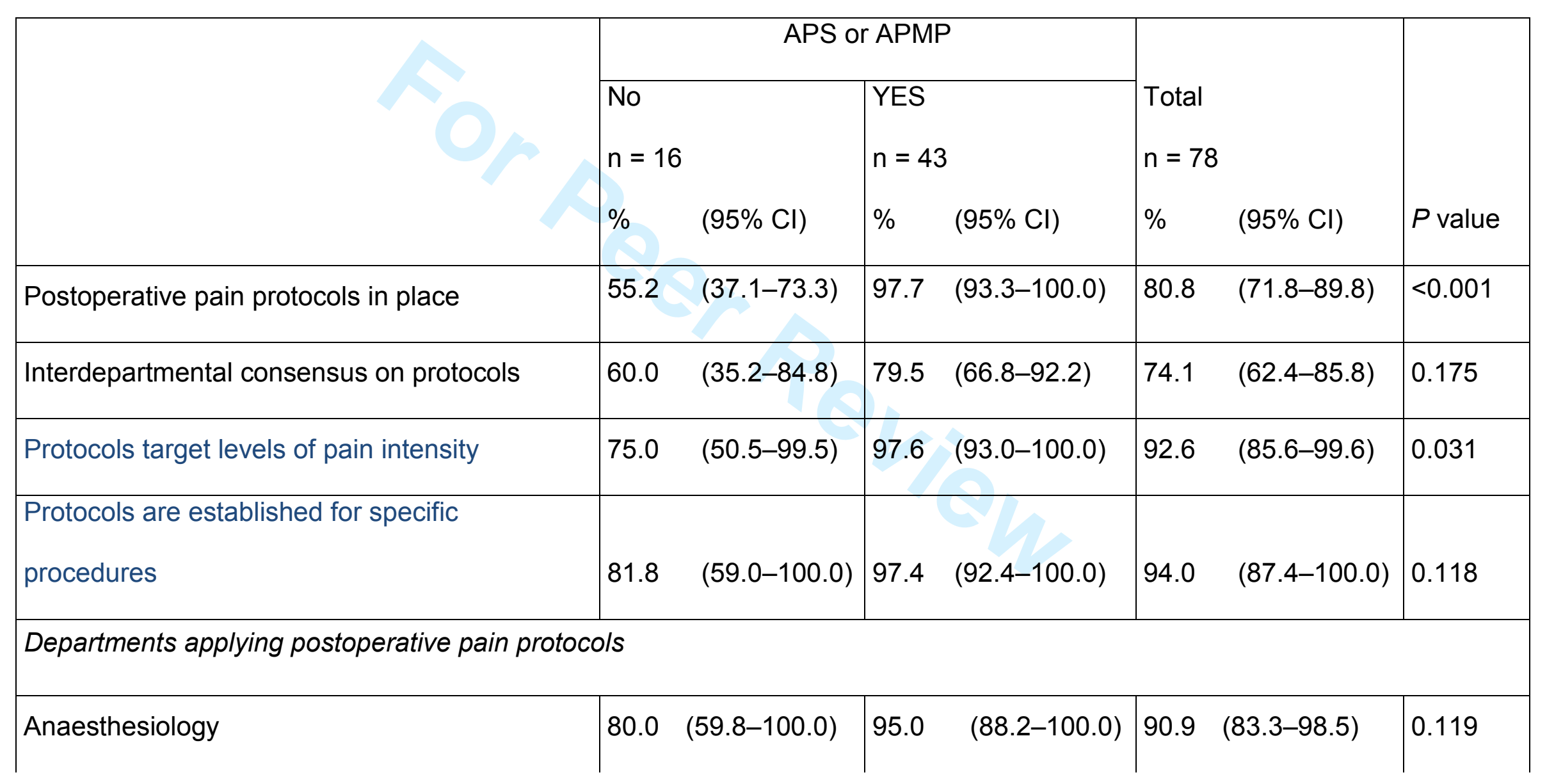




\begin{tabular}{|c|c|c|c|c|c|}
\hline Surgery & $13.3 \quad(0.0-30.5)$ & 16.3 & $(5.3-27.3)$ & $15.5 \quad(6.2-24.8)$ & $>0.999$ \\
\hline Postoperative pain service & $6.2 \quad(0.0-18.0)$ & 35.7 & $(21.2-50.2)$ & $27.6 \quad(16.1-39.1)$ & 0.045 \\
\hline Other & 0.0 & 0.0 & & 0.0 & \\
\hline
\end{tabular}


Table 5 Evaluation and recording of postoperative pain in hospitals with $\geq 200$ beds

\begin{tabular}{|c|c|c|c|c|}
\hline & \multicolumn{2}{|l|}{ APS or APMP } & \multirow[b]{2}{*}{$\begin{array}{l}\text { Total } \\
n=78\end{array}$} & \multirow[b]{2}{*}{$P$ value } \\
\hline & \begin{tabular}{|l} 
No \\
$n=33$ \\
$\% \quad(95 \% \mathrm{Cl})$
\end{tabular} & $\begin{array}{l}\text { Yes } \\
n=45^{*} \\
\% \quad(95 \% \mathrm{Cl})\end{array}$ & & \\
\hline Postoperative pain assessment is recorded & $42.4 \quad(26.6-61.0)$ & $93.3 \quad(85.4-100.0)$ & $72.0 \quad(61.8-82.2)$ & $<0.001$ \\
\hline Chart contains a place for pain assessment & $46.7 \quad(28.8-64.6)$ & $78.6 \quad(66.2-91.0)$ & $65.3 \quad(54.3-76.3)$ & 0.005 \\
\hline $\begin{array}{l}\text { Interdepartmental consensus on a } \\
\text { pain assessment scale }\end{array}$ & $41.9 \quad(23.5-59.3)$ & $92.5 \quad(84.3-100.0)$ & $70.4 \quad(59.8-81.0)$ & $<0.001$ \\
\hline Postoperative pain assessment is routine & $25.8 \quad(10.4-41.2)$ & $73.2 \quad(59.6-86.8)$ & $52.8 \quad(41.3-64.3)$ & $<0.001$ \\
\hline \multicolumn{4}{|c|}{ Where postoperative pain assessment is recorded } & 0.008 \\
\hline Patient's chart, with vital constants & $85.7 \quad(67.4-100-0)$ & $45.0(29.6-60.4)$ & $55.6 \quad(42.3-68.9)$ & \\
\hline
\end{tabular}




\begin{tabular}{|c|c|c|c|c|c|}
\hline On a specific data sheet for pain & $14.3 \quad(0.0-32.6)$ & $55.0(39.6-70.4)$ & 44.4 & $(31.1-57.7)$ & \\
\hline Digital recording & & & & & 0.019 \\
\hline No & $66.7(40.0-93.4)$ & $27.5 \quad(13.7-41.3)$ & 36.5 & $(23.4-49.6)$ & \\
\hline$\sqrt{2}+2$ & $33.3 \quad(6.6-60.0)$ & $72.5 \quad(58.7-86.3)$ & 63.5 & $(50.4-76.6)$ & \\
\hline Assessment intervals & & & & & $0.506^{*}$ \\
\hline Every $4 \mathrm{~h}$ & $0.0 \quad(-)$ & $6.7 \quad(0.0-15.6)$ & 5.7 & $(0.0-13.4)$ & \\
\hline Every $6 \mathrm{~h}$ & $0.0 \quad(-)$ & $3.3 \quad(0.0-9.7)$ & 2.9 & $(0.0-8.5)$ & \\
\hline Every $8 \mathrm{~h}$ & $0.0 \quad(-)$ & $66.7 \quad(49.8-83.6)$ & 71.4 & $(56.4-86.4)$ & \\
\hline Every $12 \mathrm{~h}$ & $0.0 \quad(-)$ & $0.0 \quad(-)$ & 0.0 & $(-)$ & \\
\hline Every $24 \mathrm{~h}$ & $0.0 \quad(-)$ & $23.3 \quad(8.2-38.4)$ & 20.0 & $(6.7-33.3)$ & \\
\hline Pain management data are analysed & $15.4 \quad(1.5-29.3)$ & $52.8^{*}(36.5-69.1)$ & 37.1 & $(25.1-49.1)$ & 0.003 \\
\hline Improvement planning is based on analysis & $23.1 \quad(6.9-39.3)$ & $75.0 \quad(60.9-89.1)$ & 53.2 & $(40.8-65.6)$ & $<0.001$ \\
\hline
\end{tabular}


of postoperative pain records

* One anomalous result was discarded for a hospital sending two different responses for

Q22 (appendix); for this item only, therefore, $\mathrm{n}=44$.

Abbreviations: APS = acute pain service; APMP = acute pain management programme;

$\mathrm{Cl}=$ confidence interval. 
Table 6 Training in postoperative pain management in hospitals with $\geq 200$ beds, \% ( $\left.\mathrm{Cl}_{95}\right)$

\begin{tabular}{|c|c|c|c|c|}
\hline & \multicolumn{2}{|l|}{ APS or APMP } & \multirow[b]{2}{*}{$\begin{array}{l}\text { Total } \\
n=78\end{array}$} & \multirow[b]{3}{*}{$P$ value } \\
\hline & \multirow{2}{*}{$\begin{array}{l}\text { No } \\
n=33 \\
\% \quad(95 \% \mathrm{Cl})\end{array}$} & \multirow{2}{*}{$\begin{array}{l}\text { Yes } \\
n=45 \\
\% \quad(95 \% \mathrm{Cl})\end{array}$} & & \\
\hline & & & $\% \quad(95 \% \mathrm{Cl})$ & \\
\hline Meetings or courses in postoperative pain management & $26.9(9.9-43.9)$ & $73.0(58.7-87.3)$ & $54.0(41.7-66.3)$ & $<0.001$ \\
\hline \multicolumn{5}{|l|}{ Targeted participants } \\
\hline Anaesthetists & $0.0 \quad(-)$ & $84.0(69.6-98.4)$ & $70.0(53.6-86.4)$ & 0.001 \\
\hline Surgical staff & $20.0(0.0-55.1)$ & $60.0(40.8-79.2)$ & $53.3(35.4-71.2)$ & 0.157 \\
\hline Anaesthesiology residents & $50.0(10.0-90.0)$ & $61.5(42.8-80.2)$ & $59.4(42.4-76.4)$ & 0.666 \\
\hline Other residents & $33.3(0.0-71.0)$ & $53.8(34.6-73.0)$ & $50.0(32.7-67.3)$ & 0.654 \\
\hline Nurses & $85.7(59.8-100.0)$ & $85.2(71.8-98.6)$ & $85.3(73.4-97.2)$ & $>0.999$ \\
\hline Other & 0.0 & 0.0 & 0.0 & \\
\hline
\end{tabular}




\begin{tabular}{|c|c|c|c|c|}
\hline Anaesthesiology department staff & $40.0(0.0-82.9)$ & $66.7(48.9-84.5)$ & $62.5(45.7-79.3)$ & 0.338 \\
\hline Acute pain unit staff & $14.3(0.0-40.2)$ & $66.7(48.9-84.5)$ & $55.9(39.2-72.6)$ & 0.028 \\
\hline Chronic pain unit staff & $50.0(10.0-90.0)$ & $18.5(3.9-33.1)$ & $24.2(9.6-38.8)$ & 0.137 \\
\hline Other & $28.6(0.0-62.1)$ & $11.1(0.0-22.9)$ & $14.7(2.8-26.6)$ & 0.268 \\
\hline \multicolumn{5}{|l|}{ Continuing education accreditation } \\
\hline Yes & $60.0(17.1-100.0)$ & $69.2(51.5-86.9)$ & $67.7(51.2-84.2)$ & $>0.999$ \\
\hline
\end{tabular}

Abbreviations: APS = acute pain service; APMP = acute pain management programme; $\mathrm{Cl}=$ confidence interval. 
Table 7 Overall assessment of acute postoperative pain management in hospitals with $\geq 200$ beds

\begin{tabular}{|c|c|c|c|c|}
\hline & APS or APMP & & & \\
\hline & $\begin{array}{l}\text { No } \\
n=33 \\
\% \quad(95 \% \mathrm{Cl})\end{array}$ & $\begin{array}{l}\text { Yes } \\
n=45 \\
\% \quad(95 \% \mathrm{Cl})\end{array}$ & $\begin{array}{l}\text { Total } \\
n=78 \\
\% \quad(95 \% \mathrm{Cl})\end{array}$ & $P$ value \\
\hline Very poorly managed & $19.0(2.2-35.8)$ & $5.7 \quad(0.0-13.4)$ & $10.7(2.6-18.8)$ & 0.001 \\
\hline Poorly managed & $33.3(13.1-53.5)$ & $8.6 \quad(0.0-17.9)$ & $17.9(7.9-27.9)$ & \\
\hline Neither poorly nor well managed & $38.1(17.3-58.9)$ & $17.1(4.6-29.6)$ & $25.0(13.7-36.3)$ & \\
\hline Well managed & $9.5 \quad(0.0-22.0)$ & $48.6(32.0-65.2)$ & 33.9 (21.5-46.3) & \\
\hline Very well managed & 0.0 & $20.0(6.7-33.3)$ & $12.5(3.8-21.2)$ & \\
\hline
\end{tabular}

Abbreviations: APS = acute pain service; APMP = acute pain management programme; $\mathrm{Cl}=$ confidence interval . 


\section{References}

1. Anonymus. Postoperative pain [Editorial]. Anaesth Intensive Care 1976; 4:95.

2. Chou R, Gordon DB, de Leon-Casasola OA, Rosenberg JM, Bickler S, Brennan T, Carter T, Cassidy CL, Chittenden EH, Degenhardt E, Griffith S, Manworren R, McCarberg B, Montgomery R, Murphy J, Perkal MF, Suresh S, Sluka K, Strassels S, Thirlby R, Viscusi E, Walco GA, Warner L, Weisman SJ, Wu CL. Management of Postoperative Pain: A Clinical Practice Guideline From the American Pain Society, the American Society of Regional Anesthesia and Pain Medicine, and the American Society of Anesthesiologists' Committee on Regional Anesthesia, Executive Committee, and Administrative Council. J Pain 2016; 17(2):131-57.

3. Joint Commission on Accreditation of Healthcare Organizations. Pain assessment and management standards-Hospitals. Comprehensive Accreditation Manual for Hospitals. The official Handbook (CAMH). Oakbrook Terrace, IL: Joint Commission on Accreditation of Healthcare Organizations, 2001.

4. Brasseur L, Larue F, Charpak Y. Postoperative analgesia in France. A study of the prescribing doctor's approach. Presse Med 1992; 21:463-7.

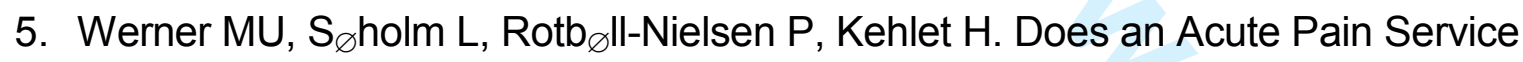
Improve Postoperative Outcome? Anesth Analg 2002; 95:1361-72.

6. Stamer UM, Mpasios N, Stüber F, Maier C. A survey of acute pain services in Germany and a Discusión of international survey data. Reg Anesth Pain Med 2002; 27:125-31.

7. Powell AE, Davies HT, Bannister J, Macrae WA. Rhetoric and reality on acute pain services in the UK: a national postal questionnaire survey. Br J Anaesth 2004; 92:68993. 
8. Van Aken H, Burkle H. Postoperative acute pain therapy: from acute pain service to acute pain program. Anasthesiol Intensivmed Noffallmed Schmerzther 2007; 42:1-20.

9. Schwenk ES, Baratta JL, Gandhi K, Viscusi ER. Setting up an acute pain management service. Anesthesiol Clin 2014; 32:893-910.

10. Powell AE, Davies HT, Bannister J, Macrae WA. Understanding the challenges of service change - learning from acute pain services in the UK. J R Soc Med 2009; 102:62-8.

11. Erlenwein J, Koschwitz R, Pauli-Magnus D, Quintel M, Meissner W, Petzke F, Stamer UM. A follow-up on Acute Pain Services in Germany compared to international survey data. Eur J Pain 2016; 20(6):874-83.

12. van Boekel RL, Steegers MA, Verbeek-van Noord I, van der Sande R, Vissers KC. Acute Pain Services and Postsurgical Pain Management in the Netherlands: A Survey. Pain Pract 2015; 15(5):447-54.

13. Puig MM, Montes A, Marrugat J. Management of postoperative pain in Spain. Acta Anaesthesiol Scand 2001; 45:465-70.

14. Catalogue of Spanish hospitals 2011. Spanish Ministry of Health, Social Policies, and Equality 2010. (cited 2016 Aug 8) Available from: http://www.msssi.gob.es/ciudadanos/prestaciones/centrosServiciosSNS/hospitales/d ocs/CNH2011.pdf.

15. Fletcher D, Fermanian C, Mardaye A, Aegerter P. A patient-based national survey on postoperative pain management in France reveals significant achievements and persistent challenges. Pain 2008; 137(2):441-51. 
16. Nasir D, Howard JE, Joshi GP, Hill GE. A survey of acute pain service structure and function in United States hospitals. Pain Res Treat 2011; 2011: 934932.

17. Duncan F, Day R, Haigh C, Gill S, Nightingale J, O'Neill O, Counsell D, NIPPS Group. First steps toward understanding the variability in acute pain service provisión and the quality of pain relief in everyday practice across the United Kingdom. Pain Med 2014; 15:142-53.

18. Goldstein DH, VanDenKerkhof EG, Blaine WC. Acute pain management services have progressed, albeit insufficiently in Canadian academic hospitals. Can J Anaesth $2004 ; 51: 231-5$.

19. Nielsen PR, Christensen PA, Meyhoff CS, Werner MU. Post-operative pain treatment in Denmark from 2000 to 2009: a nationalwide sequential survey on organizational aspects. Acta Anaesthesiol Scand 2012; 56:686-94.

20. Fermanian C, Mardaye A, Aegerter P. A patient-based national survey on postoperative pain management in France reveals significant achievements and persistent challenges, Pain 2008; 137(2):441-51.

21. Gerbershagen HJ, Aduckathil S, van Wijck AJ, Peelen LM, Kalkman CJ, Meissner W. Pain intensity on the first day after surgery: a prospective cohort study comparing 179 surgical procedures. Anesthesiology 2013; 118:934-44.

22. Rawal N, Berggren L. Organization of acute pain services: a low-cost model. Pain $1994 ; 57: 112-23$.

23. Meissner W, Ulrich K, Zwacka S. Benchmarking as a tol of continuous quality improvement in postoperative pain management. Eur J Anaesthesiol 2016; 23:142-8. 
24. De Kock M. Expanding our Horizons. Transition of Acute Postoperative Pain to Persistent Pain and Establishment of Chronic Postsurgical Pain Services. Anesthesiology 2009; 111(3):461-3.

25. Fletcher D, Stamer UM, Pogatzki-Zahn E, Zaslansky R, Tanase NV, Perruchoud C, Kranke P, Komann M, Lehman T, Meissner W; euCPSP group for the Clinical Trial Network group of the European Society of Anaesthesiology. Chronic postsurgical pain in Europe: An observational study. Eur J Anaesthesiol 2015;32(10):725-34.

26. Althaus A, Hinrischs-Rocker A, Chapman R, Arránz Becker O, Lefering R, Simanski C, Weber F, Moser KH, Joppich R, Trojan S, Gutzeit N, Neugebauer E. Development of a risk index for the prediction of chronic post-surgical pain. Eur J Pain 2012:16:901-10.

27. Müller-Schwefe G, Jaksch W, Morlion B, et al. Make a CHANGE: optimizing communication and pain management decisions. Curr Med Res Opin 2011;27:481-8.

28. Frigon C, Loetwiriyakul W, Ranger M, Otis A. An acute pain service improves postoperative pain management for children undergoing selective dorsal rhizotomy. Paediatr Anaesth 2009; 19:1213-9.

29. Schwenk ES, Baratta JL, Gandhi K, Viscusi ER. Setting up an acute pain mangement service. Anesthesiol Clin 2014; 32(4):893-910.

30. Allvin R, Brasseur L, Crul B, Dahl J, Lehmann K, Nicosia F, Puig MM, Rawal N, Richards S, Wheatley R. European Minimum Standars for the Management of Postoperative Pain. EuroPain Task Force. UK: Pegasus Healthcare Intl, 1998.

31. Neugebauer EA, Wilkinson RC, Kehlet H, Schug SA; PROSPECT Working Group. PROSPECT: a practical method for formulating evidence-based expert 
recommendations for the management of postoperative pain. Surg Endosc 2007; 21(7):1047-53.

32. Varadhan KK, Neal KR, Dejong CHC, Fearon KC, Ljunqqvist O, Lobo DN. The enhanced recovery after surgery (ERAS) pathway for patients undergoing major elective open colorectal surgery: a meta-analysis of randomized controlled trials. Clin Nutr 2010;29:434-40.

33. Benhamou D, Berti M, Brodner G, De Andres J, Draisci G, Moreno-Azcoita M, Neugebauer EA, Schwenk W, Torres LM. Postoperative Analgesic Therapy Observational Survey (PATHOS): a practice pattern study in 7 central/southern European countries. Pain 2008;136:134-41.

34. Gunningberg L, Idvall E. The quality of postoperative pain management from the perspectives of patients, nurses and patient records. J Nurs Manag 2007;15(7):75666.

35. Coluzzi F, Savoia G, Paoletti F, Constantini A, Mattia C. Postoperative pain survey in Italy (POPSI): a snapshot of current national practices. Minerva Anesthesiol 2009; 75:622-31.

36. Harmer M, Davies KA. The effect of education, assessment and a standardised prescription on postoperative pain management. The value of clinical audit in the establishment of acute pain services. Anaesthesia 1998; 53(5):424-30.

37. Tamches E, Buclin T, Hugli O, Decosterd I, Blanc C, Mouhsine E, Give, JC, Yersin B. Acute pain in adults admitted to the emergency room: development and implementation of abbreviated guidelines. Swiss Med Wkly 2007;137:223-7. 
38. Abramson JH, Abramson ZH eds. Survey methods in community medicine. Epidemiological research, programme evaluation, clinical trials. Churchill Livingstone, London: 1999.

39. Kelley K, Clark B, Brown V, Sitzia J. Good practice in the conduct and reporting of survey research. Int J Qual Health Care 2003;15(3):261-6. 
Appendix: National Survey on Postoperative Pain Management

Coordinated by the Acute Pain Group of the Spanish Pain Society (SED)

\section{Questionnaire on Postoperative Pain Management}

Before answering the questions, please provide us with information about your hospital. The survey is anonymous, so we will need your answers to these first few questions in order to stratify the responses for later analysis.

Q1. What province is your hospital located in? (Drop-down list of all Spanish provinces in alphabetical order.)

Q2. Please identify your hospital by name so we can extract information from the Spanish National Catalogue of Hospitals. (Drop-down list of hospitals by province.)

Q3. How many beds are available in your hospital?

1. Fewer than 200

2. Between 200 and 600

3. More than 600

Q4. Estimate as best you can the number of operations per week in the following hospital-use categories:
1. In-patient surgery
2. Major outpatient surgery
3. Minor outpatient surgery

Q5. Estimate as best you can the number of operations per week in each of the following surgical categories:

1. General

2. Orthopaedic/trauma

3. Cardiac

4. Plastic

5. Thoracic

6. Vascular

7.Gynaecologic/obstetric

8.Maxillofacial

9.Neurosurgery

10. Ear-nose-throat

11. Urology

12. Other

Q6. Indicate your hospital's relationship to the national health service (NHS):

1. Public (NHS-owned hospital) 
2. Private hospital affiliated with the NHS

3. Private, unaffiliated with the NHS

Q7. Indicate your hospital's residency training activity:

1. University affiliated teaching hospital giving postgraduate residency training (at least in anaesthesiology, general surgery, orthopaedic/trauma surgery)

2. University affiliated teaching hospital without residency training programs

3. Non-teaching hospital

Q8. What department of the hospital do you work in currently?

1. Anaesthesiology department

2. Pain clinic

3. Acute pain service

4. Other. Please specify. (Free-text field)

Q9. Does your hospital have an acute pain service (APS) or a hospital-wide acute pain management program (APMP)?

1. Yes (Go to Q9b.)

2. No

Q9b. Indicate the level of cover your APS or hospital-wide APMP provides:

1. Treats all surgical patients

2. Treats most surgical patients $(>70 \%)$

3. Treats a few surgical patients $(<20 \%)$

4. Treats patients with acute nonsurgical pain

Q10. What service or department takes charge of pain treatment in your hospital?

1. Anaesthesiology department

2. Acute pain service (APS) or hospital-wide acute pain management programme (APMP)

3. Chronic pain clinic

Q11. Does your hospital's acute pain service (APS) or hospital-wide acute pain management programme (APMP) manage postoperative pain for all surgical patients or only some?

1. All

2. Only some

Q12. Does your hospital employ staff specifically for the management of postoperative pain?

1. Yes, full-time physicians

2. Yes, part-time physicians

3. Yes, full-time nurses

4. Yes, part-time nurses

5. No

6. Other situations Please specify. (Free-text field)

Q13. Are you directly involved with the management of postoperative pain?

1. Yes 
2. No

The next questions are about providing patients with information about postoperative pain and assessing their pain.

Q14. Does your hospital provide patients with information about postoperative pain before surgery?

1. Yes (Go to Q14b and Q14c.)

2. No

Q14b. If you answered yes, who usually provides this information?

1. An anaesthesiologist

2. A surgeon

3. A ward nurse

4. Other. Please specify. (Free-text field)

Q14c. When is this information given?

1. During a visit with the surgeon

2. During the preanaesthesia visit

3. On the ward

4. Other. Please specify. (Free-text field)

Q15. Does the patient's chart (on paper or digitised) provide a specific space for recording postoperative pain as the fifth vital constant?

1. Yes

2. No

Q16. Is there hospital-wide consensus on which scales to use for assessing postoperative pain?

1. Yes (Go to Q16b.)

2. No

Q16b. If you answered yes, indicate which type of scale is being used.

1. Categorical scale (descriptive terms)

2. Verbal numerical scale (VNS)

3. Visual analog scale (VAS)

4. Face scale. If a face scale is used, who is it used with? (Drop-down list: Children. The elderly.

5. Other. Please specify. (Free-text field)

Q17. Does the hospital provide scales for assessing pain?

1. Yes

2. No

Q18. Is postoperative pain assessed at specified intervals?

1. Yes (Go to Q18b.)

2. No 
Q18b. If you answered yes, how often is pain assessed?

1. Every 4 hours

2. Every 6 hours

3. Every 8 hours

4. Every 12 hours

5. Every 24 hours

Q19. After postoperative pain is assessed, is the assessment recorded?

1. Yes (Go to Q19b-Q19e.)

2. No

Q19b. If you answered yes, where is the pain assessment recorded?

1. In the patient's chart, along with vital constants.

2. In a specific, separate document

Q19c. Is the recording of postoperative pain digital (computerised)?

1. Yes (Go to Q19d.)

2. No.

Q19d. Is there a pain scale threshold that obliges the administration of a rescue analgesic and/or notification of a physician responsible for the case (surgeon/anaesthesiologist/acute pain service staff physician)?

1. Yes (Go to Q19e.)

2. No

Q19e If you answered yes, what is that pain scale threshold? (Drop-down list of 0 to 10.)

Q20 Does your hospital have protocols to guide the treatment of postoperative pain?

1. Yes (Go to Q20b-Q20h.)

2. No

Q20b. If you answered yes, are the protocols the result of consensus between departments?

1. Yes (Please indicate which departments.) (Free-text field) (Go to Q20b'.)

2. No

Q20b'. Is the department of anaesthesia responsible for developing the protocols?

1. Yes

2. No

Q20c. Are the protocols classified according to level of pain intensity foreseen (e.g., mild, moderate, intense)?

1. Yes 


\section{No}

Q20d. Does your hospital designate specific pain protocols for certain surgical procedures?

1. Yes. Please specify which ones. (Free-text field)

2. No

Q20e. Which department is responsible for implementing any procedure-specific protocols at the end of surgery?

1. Anaesthesiology

2. Surgery

3. Pain clinic

4. Other. Please specify. (Free-text field)

Q20f. Which department prescribes analgesic treatments specified by the protocol for use on the ward for acute postoperative or nonsurgical pain?

1. Anaesthesiology

2. Surgery

3. Pain clinic

4. Whichever department admitted the patient

5. Other (Please specify.)

Q20g. Please specify the analgesic treatments used in each category. (Free-text field)

Oral medication. (Free-text field)

Intramuscular medication. (Free-text field)

Subcutaneous medication. (Free-text field)

Intravenous medication:

Bolus doses. (Free-text field)

Patient controlled continuous analgesia. (Free-text field)

Plexus nerve analgesia:

Bolus doses. (Free-text field)

Continuous analgesia. (Free-text field)

Epidural analgesia:

Bolus doses. (Free-text field)

Continuous analgesia. (Free-text field)

Patient controlled analgesia. (Free-text field)

Epidural analgesia:

Local anaesthetics. (Free-text field)

Opioids. (Free-text field)

Combined. (Free-text field)

Q21. Does your hospital analyse recorded information on patients' postoperative pain in order to plan improvements?

1. Yes

2. No 
Q22. Does your hospital analyse any quality-of-care indicators specific to pain management?

1. Yes

2. No

Q23. Does your hospital convene meetings or organise training courses on postoperative pain?

1. Yes (Go to Q23b-d.)

2. No

Q23b. Which staff members attend the courses target or who is convened to come to the meetings?

1. Anaesthesiologists

2. Surgeons

3. Anaesthesiology residents

4. Any residents

5. Nurses

6. Other. Please specify. (Free-text field)

Q23c. Who provides instruction for the courses or meetings?

1. Anaesthesiologists

2. Acute pain service staff

3. Chronic pain clinic staff

4. Other. Please specify. (Free-text field)

Q23d. Do participants in the courses or meetings receive continuing professional development credits?

1. Yes

2. No

Q24. Finally, please indicate how well you think postoperative pain is being managed in your hospital.

1. Very well

2. Well

3. Neither well nor poorly

4. Poorly

5. Very poorly 


\section{Acta Anaesthesiologica}

Scandinavica

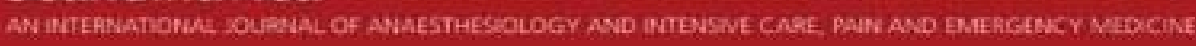

\section{Acceptance Statement}

I hereby confirm that I accept the acknowledgement statement in the paper:

"Management of postoperative pain in Spain: a nationwide survey of practice" by

“Antonio Montes, Jose Luis Aguilar, M Carmen Benito, Fernando Caba, Cesar Margarit”

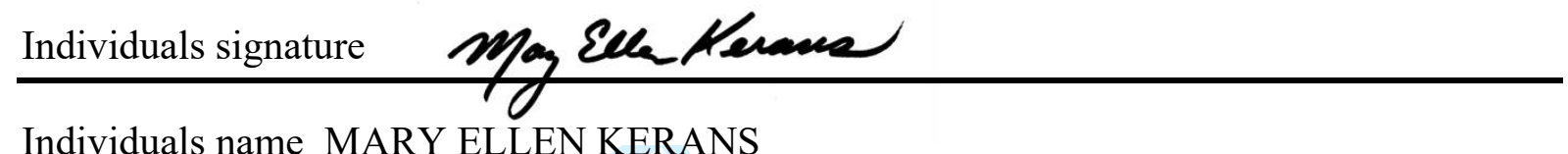

Individuals name MARY ELLEN KERANS

Date 3 November 2016 